\title{
In situ cross Linked Chitosan-Gellan Gum Polyelectrolyte Complex Based Nanogels Containing Curcumin for Delivery to Cancer Cells
}

\author{
Hitendra Shaligram Mahajan, Priyanka Pramod Patil \\ R. C. Patel Institute of Pharmaceutical Education and Research, Shirpur, Dhule, Maharashtra, INDIA
}

\begin{abstract}
Objective: The objective of this study is to prepare nanogels were prepared via ionic complexation with negatively charged gellan gum. Method: Nanogel of curcumin was prepared by in situ cross linking reaction between two oppositely charged materials by green method without use of chemical cross linking agents. The prepared nanogels were characterized by Dynamic light scattering, scanning electron microscopy, differential scanning calorimetry and X- Ray diffractometry. Results: The prepared formulation had average particle size of $226 \mathrm{~nm}$ with polydispersity index of 0.3 . The curcumin loaded nanogel demonstrated sustained release for $20 \mathrm{~h}$. The prepared nanogels were hemocompatible and cyctocompatible as revealed by hemocompatibility and MTT assay respectively. Conclusion: All results confirmed that these nanogels can be used for cancer treatment.
\end{abstract}

Key words: Nanogel, Chitosan, Gellan gum, Curcumin, Cancer.

\section{INTRODUCTION}

Chitosan, a linear polysaccharide composed of randomly distributed $\beta$-(1-4) - linked D-glucosamine (deacetylated unit) and $\mathrm{N}$-acetyl-D-glucosamine (acetylated unit). It is obtained by alkali treatment of crustacean shells. ${ }^{1}$ Chitosan is a hydrophilic, biocompatible and biodegradable polymer of low toxicity. The cationic polyelectrolyte nature of chitosan could provide a strong electrostatic interaction with negatively charged materials. ${ }^{2}$

Gellan gum, a linear anionic microbial polysaccharide that is secreted by the nonpathogenic strain Sphingomonas paucimobilis (formerly known as Pseudomonas elodea). Gellan is hydrophilic, non toxic, biocompatible and biodegradable polysaccharide suitable for application in pharmaceutical technology. Gellan is capable of gelation in the presence of mono and divalent ions; this property makes this polysaccharide suitable as a gelling agent in foods and for several other applications. Gellan gum forms transparent gels which are heat-resistant, and the gel strength is less dependent on $\mathrm{pH}$ in comparison with other polysaccharide gels. ${ }^{3,4}$ Nanogels are defined as a cross-linked polymeric network capable of swelling in a good solvent. ${ }^{5}$ Nanogels demonstrate great potential in smart, controlled, and regulated applications due to their environmentally responsive character. Specifically, they increasingly became recognized as the carrier for therapeutic drugs and diagnostic agents. The porous polymer network structure of synthetic nanogels offers an ideal reservoir for loading drugs, protecting them from environmental degradation and hazards. ${ }^{6,7}$ Chitosan shows the ability to form hydrogels able to control the rate of drug release from the delivery system as well as protect the drug from chemical and enzymatic degradation in the administration site. In particular, when chitosan is
Submission Date: 15-12-2016; Revision Date: 24-01-2017; Accepted Date: 15-02-2017

DOI: $10.5530 /$ ijper.51.2s.48 Correspondence: Dr. Hitendra S Mahajan, R. C. Patel Institute of Pharmaceutical Education \& Research, Near Karvand Naka, Shirpur-425405, Dist: Dhule, Maharashtra, INDIA.

Fax: 91-2563255180

Phone: 91-9423487043

E-mail: hsmahajan@rediffmail.com

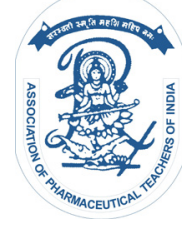

www.ijper.org 
cross-linked or complexed with an oppositely charged polyelectrolyte, a three-dimensional network is formed in which the drug can be incorporated in order to control its release. The selection of suitable conditions during chitosan / polyanion complexes is a novel strategy for the realization of pharmaceutical systems with interesting functional properties (drug loading ability, stability and drug delivery) and allow the modulation of the delivery system behaviour at the administration or therapeutic site. Chitosan-based polyelectrolyte complexes e.g. hyaluronic acid, alginic acid, pectin and gelatin have been developed for local or systemic administration of drugs. The choice polyelectrolyte complexes, with specific functional properties such as good absorption enhancing ability as well as good control of drug release, associated with the use of suitable preparative methodologies, has led to the development of innovative systems for the delivery of drugs with poor pharmacokinetic profile. We hypothesized that gellan gum could provide superior functional properties of polyelectrolyte. Thus in present study, chitosan and gellan gum were selected to produce polyelectrolyte complexes, with the aim of investigating their possible application for drug delivery.

Chitosan and gellan gum nanogel has formed as result of electrostatic interaction between chitosan and gellan gum. In present study the curcumin loaded nanogel based on chitosan and gellan gum is first time reported by us that are prepared by green production process uses only water. The chemically cross linked nanogels formed by covalent interactions are stable and capable of maintaining their robust structure under all environmental condition unless a labile functional group has been attached to the network. Chemically cross linked nanogels size range between 100 to $1000 \mathrm{~nm} .{ }^{8}$ Various approaches have been currently running to cure the cancer through polymeric nanoparticles. All the nanoparticles that are FDA approved for cancer therapy or in advanced clinical testing rely on the enhanced permeability and retention (EPR) mechanism, which enables passive targeting through the disorganized and leaky vasculature and reduced lymphatic drainage associated with tumors. ${ }^{9}$ The advance drug delivery approaches such developing smart carrier and targeting them at specific tissues or cells is requiring in effective management of cancer.

Curcumin is the active constituent of turmeric (curcuma longa) and also been found to be beneficial in all three stages of carcinogenesis but it is not widely used because of its poor aqueous solubility. Curcumin also faces pharmacokinetic problems such as poor absorption as well as bioavailability hence in effective in cancer treatment. Thus, the specific objective of our study is to develop curcumin loaded chitosan- gellan gum nanogels for intravenous administration and to analyze their efficacy against the cancer cell line.

\section{MATERIALS AND METHODS}

\section{Materials}

Chitosan (degree of deacetylation $\sim 79 \%$, Low mol wt ,Viscosity 200-800 cP, 1 wt. \% in 1\% acetic acid) from Sigma Aldrich, India, Gellan gum (Low acyl content, mol. wt $>70,000$ Daltons) obtained as gift sample from Burzin and Leons, CPKelco division of the Monsanto Company, USA. Curcumin (95\% purity, Product no. 0000155108) purchased from Hi Media Lab. Pvt. Ltd, Mumbai, India.

\section{Method}

\section{Preparation of chitosan- gellan gum nanogel}

Chitosan $0.85 \% \mathrm{w} / \mathrm{v}$ solution was prepared in acetic acid and $0.04 \% \mathrm{w} / \mathrm{v}$ gellan gum solution was prepared in distilled water. Both the solutions were filtrated by vaccum filter using $0.22 \mu \mathrm{m}$ filter paper. Chitosan solution added slowly to gellan gum under agitation at room temperature for $3 \mathrm{~h}$. The $\mathrm{pH}$ of the resultant solution was about 4.3 and was adjusted to $\mathrm{pH}$ 5.4. The mixture was heated at $80^{\circ} \mathrm{C}$ for 20 min to produce homogeneously dispersed nanogel, and then the nanogels were separated by centrifugation at $45,000 \mathrm{rpm}$ for $35 \mathrm{~min}$ utes (Beckman Coulter, ultracentrifuge).

\section{Preparation of Curcumin loaded chitosan- gellan gum nanogel}

Curcumin was loaded into the nanogel along with chitosan solution and the same procedure was followed as described for chitosan - gellan gum nanogel. The curcumin loaded nanogels were separated by centrifugation at 45,000 rpm for 35 minutes (Beckman Coulter ultracentrifuge). Deposited particles were re dispersed in distilled water containing $5 \%$ mannitol as a cryoprotectant. The resultant dispersion was subjected to lyophilisation at $-75^{\circ} \mathrm{C}$ and vacuum was maintained at $76 \mathrm{~m}$ Torr (Vertis Lyophilizer).

\section{Characterization of nanogel}

The average particle size and polydispersity index was determined by photon correlation spectroscopy and Zeta potential of system was measured using Zeta sizer, (Nano ZS 90, Malvern Instrument., UK). The surface morphology of nanogel was examined by scanning electron $^{\circledR}$ microscopy (JSM 6390, Japan). To demonstrate physical state of curcumin within nanogels, differential 
scanning calorimetry (DSC, Mettler-Toledo, Zurich, Switzerland), and X-ray diffractometry (XRD, Brucker Axs, D8 Advance; Germany) analysis were performed.

\section{Entrapment efficiency, Loading efficiency and in vitro drug release studies of curcumin loaded nanogel}

Curcumin entrapment in chitosan- gellan gum nanogel formulation was determined by triturating lyophilized powder with DMSO and methanol in mortar pestle and diluted up to $10 \mathrm{ml}$ in volumetric flask. The resulting solution was centrifuged at 30,000 rpm for $20 \mathrm{~min}$ and the supernatant was collected and analyzed spectrophotometrically (UV 1700, Shimadzu, Japan) at $423 \mathrm{~nm}$. Drug content was estimated using equation $(y=0.08 x$ - 0.010) generated as result of plot of concentration vs absorbance that obeys Beer's and Lambert's law in concentration range 2 to $12 \mu \mathrm{g} / \mathrm{mL}$. Loading efficiency was calculated in relation to yield of nanogel obtained after

$$
\text { Loading efficiency }(\%)=\frac{\text { Actual amount curcumin in nanogel }}{\text { Total yield of curcumin loaded nanogel }} \times 100
$$

lyophilisation (equation- 1).

Entrapment efficiency was calculated on the basis of total amount of curcumin in nanogel to total amount of curcumin used during drug loading. ${ }^{10}$ (equation 2)

Entrapment efficiency $(\%)=\frac{\text { Total amount of curcumin in nanogel }}{\text { Total amount of curcumin }} \times 100$

In vitro curcumin release from nanogel formulation was carried out by using Franz type diffusion cell of $25 \mathrm{ml}$ capacity. Dialysis membrane (Himedia, Avg mol. weight cut off range $12000-14000 \mathrm{kDa}$ ) was used as diffusion membrane. Dialysis membrane was soaked in phosphate buffer $\mathrm{pH} 7.4$ for $24 \mathrm{~h}$ prior to experiment. Diffusion cell was filled with buffer solution and dialysis membrane was mounted on cell. The temperature was maintained at $37^{\circ} \mathrm{C} \pm 0.5^{\circ} \mathrm{C}$. After a pre-incubation time of 20 minutes, the lyophilized nanogel was placed in the donor chamber. Samples were periodically withdrawn from the receptor compartment and replaced with the same amount of fresh buffer solution, and assayed by a UV spectrophotometer at $423 \mathrm{~nm}$.

To evaluate the mechanism of drug release from the nanogel, data for the drug release was plotted in Korsmeyer-Peppas equation. The release exponent $\mathrm{n}$ and $\mathrm{K}$ value was calculated through the slope of the straight line. (3)

$$
\mathrm{M}_{\mathrm{t}} / \mathrm{M}_{\infty}=\mathrm{Kt}^{\mathrm{n}}
$$

\section{Hemolysis Assay}

The blood compatibility of the chitosan -gellan gum nanogel by hemolysis assay using human blood obtained from blood bank. In different concentrations of nanogel $(0.2,0.4,0.6,0.8,1 \mathrm{mg} / \mathrm{ml}), 0.1 \mathrm{ml}$ of anti coagulated fresh human blood was added. Positive and negative control selected for this study were PBS ( $0 \%$ hemolysis) and $0.1 \%$ of $\mathrm{Na}_{2} \mathrm{Co}_{3}$ (100\% Hemolysis). After incubation at 37 for 90 min sample were centrifuge at 4500 rpm for 5 min. Absorbance of supernant was analyzed spectrophotometrically against PBS at $545 \mathrm{~nm}^{.1}$

The percentage of haemolysis was calculated by the following equation 4 .

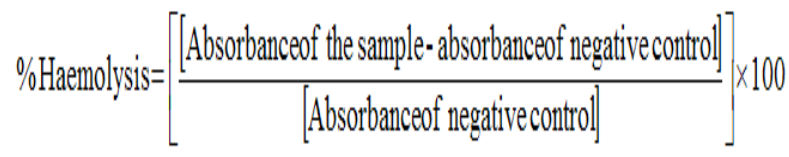

\section{Cell culture protocol}

Astrocytoma - glioblastoma cell line (U373MG) was used for this study. The cells were seeded into 96-well plates at a density of $1.0 \times 10^{4}$ cells per well and grown in Dulbecco's modified Eagle's medium (DMEM) supplemented with 10\% fetal bovine serum (FBS), 100 $\mu \mathrm{g} / \mathrm{mL}$ penicillin, $200 \mu \mathrm{g} / \mathrm{mL}$ streptomycin, $2 \mathrm{mM}$ L-glutamine; and culture was maintained in a humidified atmosphere with 5\% $\mathrm{CO}_{2}$. The cells were divided into two treatment groups, including: (a) curcumin suspension and (b)freeze dried curcumin loaded nanogel and $\%$ growth inhibition was determined by MTT assay based on mitochondrial reduction of yellow MTT tetrazolium dye to highly colored blue formazan product. The plates were incubated for $48 \mathrm{~h}$ at $37 \pm 0.5^{\circ} \mathrm{C}$ in RPMI/DMEM/MEM with 10\% FBS medium. Then the above media was replaced with $90 \mu \mathrm{L}$ of fresh serum free media and $10 \mu \mathrm{L}$ of MTT reagent $(5 \mathrm{mg} / \mathrm{mL})$ and plates were incubated at $37 \pm 0.5^{\circ} \mathrm{C}$ for $4 \mathrm{~h}$, there after the above media was replaced with $200 \mu \mathrm{L}$ of DMSO and incubated at $37 \pm 0.5^{\circ} \mathrm{C}$ for $10 \mathrm{~min}$. Further studies were carried in accordance with our previous protocol. ${ }^{12}$

\section{RESULTS AND DISCUSSION}

\section{Preparation and characterization of curcumin loaded chitosan- gellan gum nanogel}

Curcumin loaded chitosan- gellan gum nanogel was formed immediately as result of cross-linking between positively charged chitosan and negatively charged gellan gum by co- acervation reaction due to electrostatic attraction between two opposite charged polymers. However, the networks thus obtained by in situ cross- 
linking are reversible and unable to retain integrity of nanogel system. Heating at $80^{\circ} \mathrm{C}$ for 20 min leads to formation of nanogel; these nanogels have good stability and do not require any stabilizer. Curcumin loaded nanogel were obtained by addition of curcumin to chitosan solution prior to cross-linking with gellan gum.

\section{Characterization of nanogel}

The average particle size for chitosan- gellan gum nanogel was found to be $201 \mathrm{~nm}$ and $226 \mathrm{~nm}$ for curcumin loaded nanogel with polydispersity index value of 0.31 and 0.39 respectively, demonstrating uniform distribution of nanogel population. Such nano range size distribution crucial for passive tumor targeting due to enhanced permeation and retention effect as majority of solid tumors exhibit a vascular pore cut-offs between 380 and $780 \mathrm{~nm} \cdot{ }^{13}$ From SEM image of nanogel it is clear that the freeze dried nanogel have smooth surface with porous nature. Zeta potential of freeze dried curcumin loaded nanogel was found to be 5.52.The Zeta potential represents the electrical charge to the nanogel surface suggest that chitosan molecules are enriched on the particle surface. During thermal analysis curcumin showed strong endothermic peak at a temperature around $180^{\circ} \mathrm{C}$, due to its crystalline nature (Figure 2A) but nanogel formulation displayed absence of peak in this region (Figure 2B). Also in X-ray diffractometry

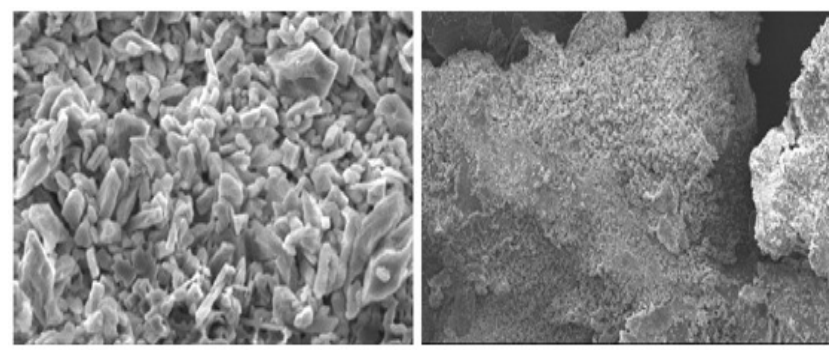

Figure 1: SEM image of freeze dried curcumin loaded nanogel

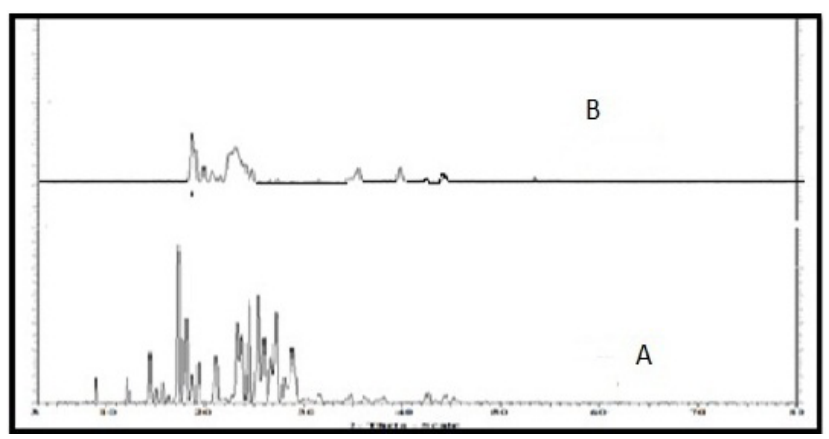

Figure 3: X- Ray Diffractogram of curcumin (A) and nanogel (B) pattern numerous crystalline peaks were observed in the $2 \theta$ range of $15-30^{\circ}$ demonstrate crystalline nature of curcumin (Figure 3A) where as there were decrease in peak intensity in nanogel formulation confirms the amorphous state of curcumin in nanogel (Figure 3B).

\section{Entrapment efficiency, Loading efficiency and drug release studies}

The loading and entrapment efficiency of curcumin loaded nanogel system was found to be $5.66 \pm 0.78 \%$ and $96.60 \pm 1.28 \%$ respectively. The higher entrapment of curcumin in nanogel structure is attributed to in situ cross linking between two polymers and addition of curcumin prior to completion of interaction. To establish the suitability of chitosan - gellan gum nanogel as delivery system for curcumin, the in vitro release was studied. The release profile of curcumin-loaded nanogel was studied using dialysis membrane. Sustained release of curcumin from nanogel was observed (Figure 4a). The corresponding plot of Korsmeyer - Peppa's equation (Figure $4 \mathrm{~b}$ ) indicated a good linearity of regression coefficient $\left(R^{2}\right)$ 0.913. The release exponent (n) of Korsmeyer-Peppa's equation was found to be 0.91 and $\mathrm{K}$ value 0.869 . The $\mathrm{n}$ value was greater than 0.5 and less than 1 indicating anomalous (non-fickian) transport mechanism suggesting curcumin release due to diffusion through swell chitosan- gellan gum matrix. ${ }^{14}$

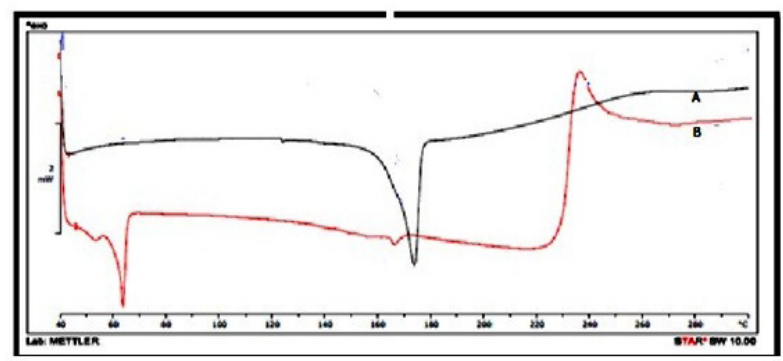

Figure 2: DSC thermaogram of curcumin (A) and nanogel (B
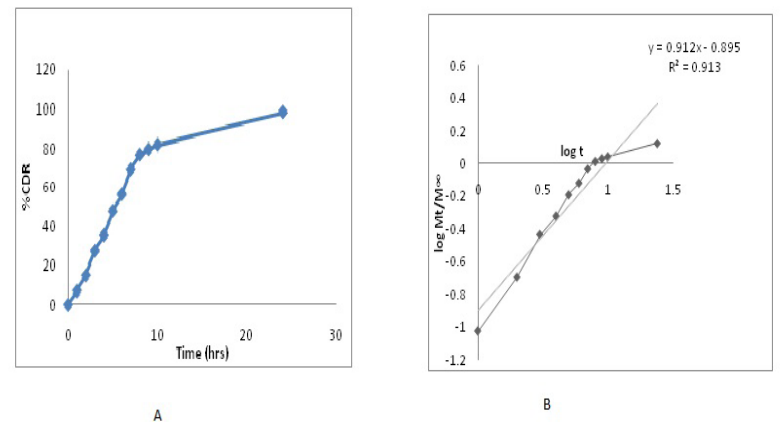

Figure 4: Curcumin release profile $(\mathrm{A})$ and release mechanism (B) 


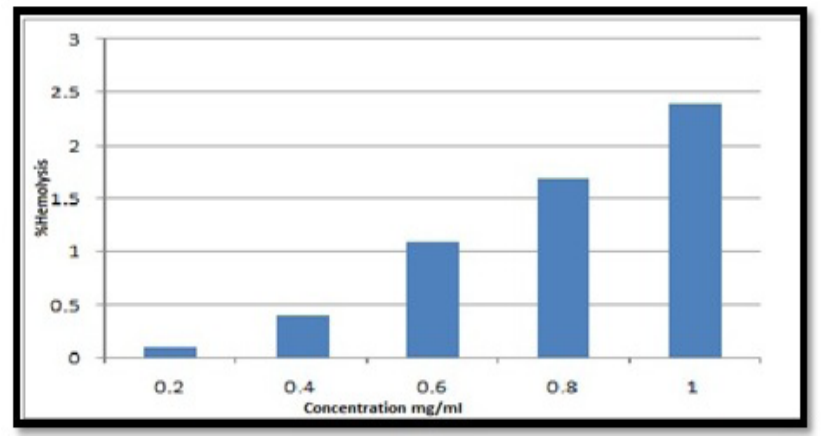

Figure 5: Hemocompatibility of nanogels

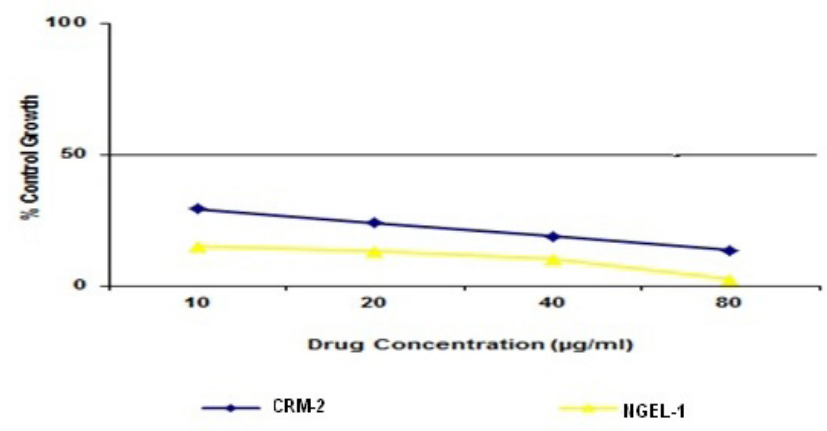

Figure 6: In vitro cytotoxicity assay

\section{Haemolysis Assay}

Haemocompatibility of the nanogels was essential as nanogels are designed for drug delivery purpose that interacts with a large fraction of blood component in the blood before distribution to individual tissue. For drug delivery purpose nanogels must show minimum interaction with components in the blood stream and should possess long-term stability. On analysis of haemolytic potential of five different concentrations of nanogels it was observed that increase in nanogel concentration leads to increase in hemolytic ratio (Figure 5). It is about to $2.5 \%$ i.e. below $5 \%$, the critical safe hemolytic ratio for bio-materials, indicated that these chitosan-gellan gum nanogel samples are compatible with blood components. ${ }^{15}$

\section{Cytotoxicity Studies}

Cytotoxicity assay of curcumin loaded nanogel was performed and compared with standard (curcumin) by cell viability testing. Figure 6 shows plot of $\%$ inhibition of astrocytoma-glioblastoma cell line (U373MG) incubated with the curcumin loaded nanogel vs curcumin. The $\mathrm{IC}_{50}$ values were found out to be 9.8 and $13.6 \mathrm{ng} / \mathrm{ml}$ for curcumin loaded nanogel and curcumin suspension respectively. Thus, curcumin loaded nanogel showed lower cellular viability demonstrating increased cytotoxicity of formulation towards cells. ${ }^{16}$

\section{CONCLUSION}

Curcumin loaded chitosan- gellan gum nanogels were prepared by in situ cross-linking between two oppositely charged polymers. The prepared nanogels subjected good stability devoid of use of external chemical cross-linkers. Prepared nanogels were characterized for particle size, zeta potential, and surface morphology. The result of particle size analysis reveals there suitability for effective tumor targeting. The prepared nanogel showed porous nature due to lyophilisation with positive zeta potential confirming dominance of chitosan on surface. The curcumin loaded nanogel showed good entrapment efficiency and sustained drug release. In vitro cytotoxicity and hemocompatability assay proved the nontoxic and hemocompatible nature of the nanogels. These results indicated that chitosan- gellan gum nanogel could be suitable carrier for curcumin in cancer treatment.

\section{ACKNOWLEDGEMENT}

Authors are thankful to Burzin and Leons, CPKelco division of the Monsanto Company (U.S.A.) for gifting gellan gum. One of the author (Dr.H.S.Mahajan) is thankful to University Grant Commission (UGC New Delhi) for Research Grant.

\section{CONFLICT OF INTEREST}

The authors report no declarations of interest

\section{ABBREVIATION USED}

FDA: Food and Drug Administration; DMSO- Dimethylsulfoxide.

\section{REFERENCES}

1. Anitha A, Divya RVV, Krishna R, Sreeja V, Selvamurugan N, Nair SV. Synthesis, characterization, cytotoxicity and antibacterial studies of chitosan,O-carboxymethyl and N, O-carboxymethyl chitosan nanoparticles. Carbohyd Polym. 2009;78(4):672-7.

2. Jayakumar R, Chennazhi KP, Muzzarelli RAA, Tamura H, NairSV, Selvamurugan N. Chitosan conjugated DNA nanoparticles in gene therapy. Carbohyd Polym. 2010;79(1);1-8.

3. Janssen PE, Lindeberg B, Sandford PA. Structural studies of gellan gum, an extra cellular polysaccharide elaborated by Pseudomonas eloda. Carbohyd Res. 1983;124(1):135-9.

4. Malcolm A, O'Neill R, Selvendran R, Morris VJ. Structure of the acidic extracellular gelling polysaccharide produced by Pseudomonas elodea. Carbohyd Res. 1983;124(1):123-33.

5. Sasaki Y, Akiyoshi K. Nanogel engineering for new nanobiomaterials: from chaperoning engineering to biomedical applications. Chemical record (New York, N.Y.) 2010;10(6):366-76.

6. Rhee Y, Mansour HM. Nanopharmaceuticals I: nanocarrier systems in drug delivery. Int J of Nanotech. 2011;8(1-2):84-114. 
Wu X, Mansour HM. Nanopharmaceuticals II: application of nanoparticles and nanocarrier systems in pharmaceutics and nanomedicine. Int $\mathrm{J}$ of Nanotech. 2011;8(1-2):115-45.

8. Gupta P, Vermani K, Garg S. Hydrogels: from controlled release to $\mathrm{pH}$ responsive drug delivery. Drug discovery today. 2002;7(10):569-79.

9. Matsumura Y, Maeda HA. New concept for macromolecular therapeutics in cancer chemotherapy: mechanism of tumoritropic accumulation of proteins and the antitumor agent smancs. Cancer Res. 1986;46(12):6387-92.

10. Anitha A, Deepagan VG, Divya R VV, Deepthy M, Nair SV, Jayakumar R. Preparation, characterization, in vitro drug release and biological studies of curcumin loaded dextran sulphate-chitosan nanoparticles. Carbohyd Polym. 2011;84(3);1158-64.

11. O'Leary RR, Watkins WD, Guess WL. Comparative chemical and toxicological evaluation of residual ethylene oxide in sterilized plastics. J Pharm Sci. 1969;58(8):1007-10.
12. Madane RG, Mahajan HS. Curcumin-loaded nanostructured lipid carriers (NLCs) for nasal administration: design, characterization, and in vivo study. Drug Del. 2014;23(4):1-9.

13. Zahr AS, Davis CA, Pishko MV. Macrophage uptake of core shell nanoparticles surface modified with poly (ethylene glycol). Langmuir. 2006; 22(19):8178-85..

14. Peppas NA. Analysis of fickian and non fickian drug release from polymers Pharm Acta Helv. 1985; 60:110-11.

15. Sarika PR, Nirmala RJ. Preparation and characterisation of gelatin-gum arabic aldehyde nanogels via inverse miniemulsion technique. Int Journal of Biol Mac. 2015;76:181-7.

16. Zhao L, Feng S. Enhanced oral bioavailability of paclitaxel formulated in vitamin E-TPGS emulsified nanoparticles of biodegradable polymers: in vitro and in vivo studies. J of Pharm Sci. 2010;99(8):3552-60.

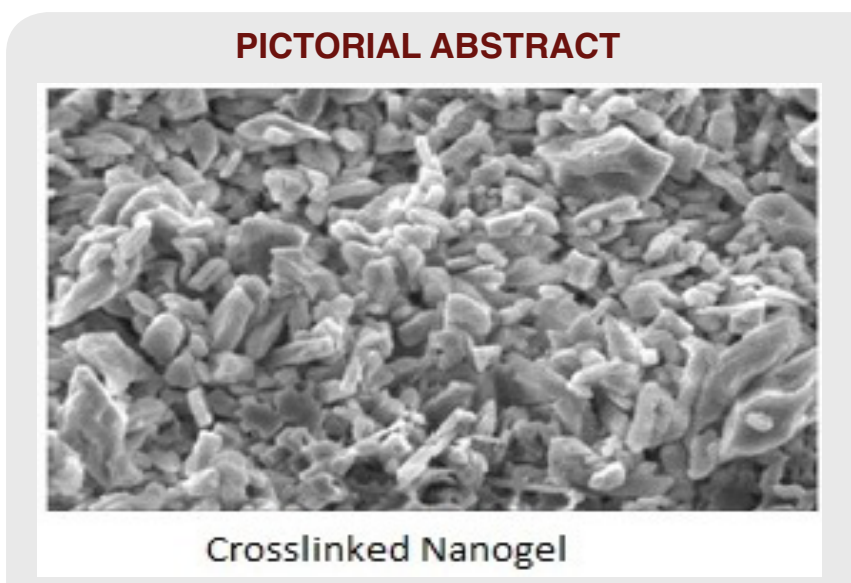

\section{SUMMARY}

Nanogels are ideal candidates for intracellular delivery into the cytoplasm of the cell. Nanogels show promise as a suitable nanomedicine carrier as compared to other nanoparticles especially in terms of drug loading. Nanogels can be prepared or synthesized even in the absence of the drug to be loaded as drug loading in nanogels can be efficiently done later on when the nanogels are swollen and equilibrated in water or biological fluid. Drug loading occurs spontaneously in nanogels. Hence the study has under taken to formulate Curcumin loaded nanogel to target cancer cell.

\section{About Authors}

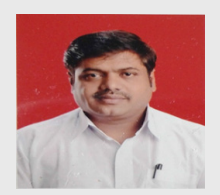

Dr.H.S.Mahajan Corresponding author, currently working as Head Dept of Pharmaceutics at R.C.Patel Institute of Pharmaceutical Education and Research Shirpur dist Dhule. He had 15 years of teaching and academic experience.

Priyanka Patil, She is post graduate student of Dept of Pharmaceutics at R.C.Patel Institute of Pharmaceutical Education and Research Shirpur dist Dhule.

Cite this article: Mahajan HS, Patil PP. In situ cross Linked Chitosan-Gellan Gum Polyelectrolyte Complex Based Nanogels Containing Curcumin for Delivery to Cancer Cells. Indian Journal of Pharmaceutical Education and Research. 2017;51(2S):S40:S5. 